\title{
Wavelet Entropy and Neural Network Based Fault Detection on A Non Radial Power System Network
}

\author{
Soumyadip Jana ${ }^{1}$, Gaurab Dutta ${ }^{1}$ \\ I'Department of Electrical Engineering, Techno India, EM 4/1 Salt Lake, Sec-V, Kolkata-700091, India)
}

\begin{abstract}
A novel application of neural network approach to classify the fault types and to identify the faulty lines in a non radial power system network is demonstrated in this paper. EMTP software has been used to simulate the transmission line model. The simulation is followed by analysis of the current waveforms using wavelet transform in the MATLAB environment. The entropy values of the current signal obtained from wavelet transform have been fed into the neural network for automatic fault type classification and faulty line identification. It has been found that only three level of decomposition of the current signal is sufficient for analysis of faults. The adaptive discrimination scheme is tested under different types of faults, such as single line to ground faults, line-to-line faults, double line to ground faults and three phase symmetrical faults for different varying fault locations and fault resistances, on a given power system model. Results of performance studies show that the proposed scheme for fault diagnosis is able to classify all the faults and to identify the exact faulty lines on the non radial power system network rapidly and correctly.
\end{abstract}

Keywords - Entropy, Faults, Probabilistic neural network, Wavelet transform

\section{INTRODUCTION}

In an electric power system Transmission networks are the important components. Faults on power system transmission lines need to be detected and located rapidly, classified correctly and cleared as fast as possible to increase system's reliability, security and delivered energy quality. In power system protection schemes classification of fault types and identification of faulty lines in a non radial system are the important issues. This can be done by detecting, localizing and classifying different fault types and identifying faulty lines. Safty and Zonkoly [1] presented a paper where EMTDC/PSCAD software has been used to detect fault types and wavelet entropy principle is implemented to analyze the current signals. Routray et al. [2] proposed a real time wavelet-fuzzy combined approach for digital relaying. Jung et al. [3] used a MATLAB [4] based simulation tool to calculate the short circuit faults in power system transmission lines. The discrete wavelet transform has been used along with the fuzzy logic system to detect the fault classification of a transmission line. Kim et al. [5] presented a fault location algorithm using the Neuro-fuzzy systems in a combined transmission lines along with underground power cables. A fault location method employing wavelet fuzzy neural network has been proposed by Chunju et al. [6]. A new one-end fault location method for overhead transmission lines embedded in a general $n$-bus interconnected system has been discussed by Eisa et al. [7]. Salim et al. [8] presented a paper in which fault location on transmission lines has been detected by impedance calculation method. Borghetti et al. [9] presented a paper based on a continuous wavelet transform for the analysis of voltage transients due to line faults, and discussed its application to fault location in power distribution systems. Magnago et al. [10] identified the fault location on the parallel transmission lines using wavelet. Saleh et al. [11] proposed a differential equation to obtain the transient and steady state condition analysis. Fault location in transmission line is presented by Ekici et al.[12]. He and Starzyk [13] proposed a novel approach for PQ disturbances classification based on thewavelet transformand self organizing learning array (SOLAR) system in the paper. Jain et al. [14] presented an extension to neural network based transmission line fault detection and classification technique for single circuit transmission line to double circuit transmission line and proposed an adaptive protection scheme for such systems by using the ANN approach. Das et al. Presented a comparison of the Fourier Transform method with Wavelet Transform method for detection and classification of faults on transmission lines. Reference [16] showed an application of artificial neural network approach to fault classification for double circuit transmission lines using superimposed sequence components of current signals. An algorithm of fault classification and faulted phase selection for a single circuit transmission line based on the initial current traveling wave is very recently proposed in [17].Ngaopitakkal et al. [18] presented identification of simultaneous faults on transmission system using wavelet transform.

In this paper, half cycle of the pre fault and half cycle of the post fault current signal has been considered and decomposed by wavelet transform up to level 3. It has been found that the entropy values of detail coefficients of level 1 and level 3 only are sufficient for the detection and classification of faults and identification of faulty lines in a non radial power system network. The entropy values have been fed into the 
neural network for automatic fault classification and faulty line identification. The proposed protection scheme is tested under different fault types, varying fault location and varying fault resistances.

\section{POWER SYSTEM MODEL}

A six bus eight line non radial power system network has been considered here. The simulation has been done in Electro Magnetic Transient Program. This program allows us to simulate the faults of any types at any location along the transmission line. In this case a $400 \mathrm{~V}, 15 \mathrm{~km}$ six bus eight line non radial power system network, as shown in figure $\mathrm{I}$, is considered. The length of each line is $15 \mathrm{~km}$. The transmission lines are constructed with 30 identical 3 phase LCC blocks of $0.5 \mathrm{~km}$ each. The voltage for the two generators is assumed to be $11 \mathrm{KV}$ and the MVA rating is $10 \mathrm{MVA}$. The rating of the power transformers are $11 \mathrm{KV} / 440 \mathrm{~V}$ and $10 \mathrm{MVA}$, each having delta-star connection. The three phase balanced load are connected at the four load buses. All types of faults are simulated at $0.5 \mathrm{~km}$ intervals in all the eight lines. The current signals are monitored from bus 1 only. The line resistance is taken $0.0585 \mathrm{ohm}$ per $\mathrm{km}$.

The fault resistance considered in this case is $10 \mathrm{ohms}$ and the total simulation time in 80 milliseconds. The sampling frequency is 2000 samples per cycle. All the different types of faults are generated after 40 milliseconds i.e. after two cycles. The technique developed in this paper mainly detects the type of faults and identifies the faulty lines in the transmission line.

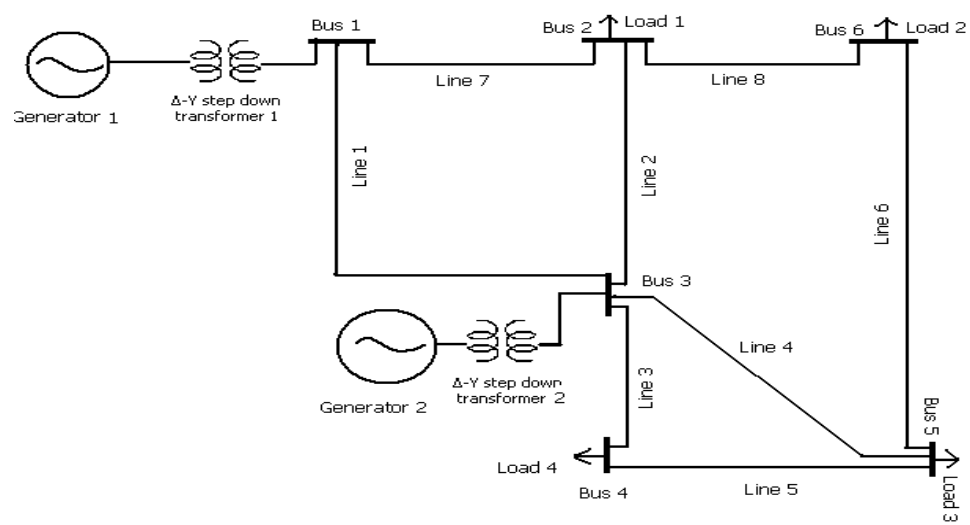

Fig I: Simulated 6bus-8line non radial power system network

EMTP model of the proposed 6bus-8line non radial power system network is shown in figure II. For classification of fault types and identification of faulty lines simulations are occurred at 240 different locations, the faulty current signals are collected and analyzed in ATP analyzer.

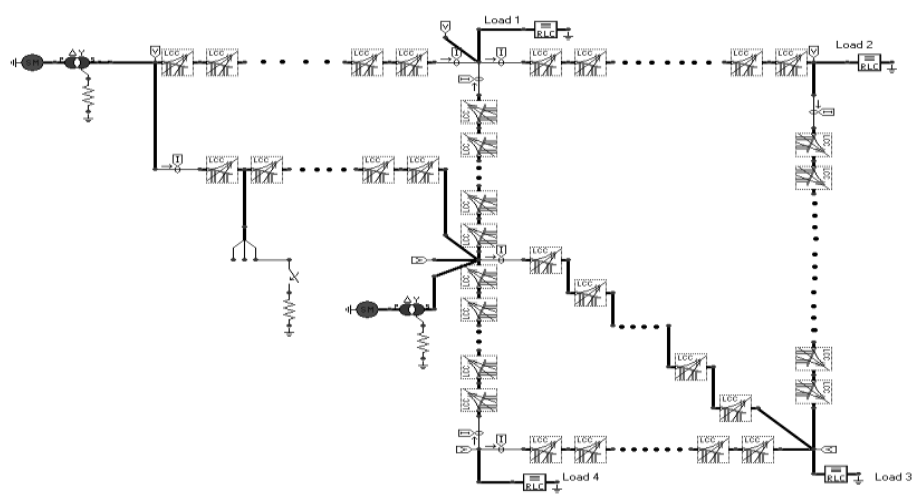

Figure II: EMTP model of 400V-15km 6bus-8line non radial power system network

III.

\section{FEATURE EXTRACTION USING WAVELET TRANSFORM}

Feature extraction can be defined as a unique process that transforms the raw signals from its original form to a new form to extract suitable information from them. The feature extraction step is crucial in an automatic classification system. This is because a classifier can operate reliably only if the features of each event are selected properly. During fault, the amplitude and frequency of the test signal will change significantly as the system change from normal state to faulty state. The Shannon entropy will change accordingly. Wavelet transformation has the ability to analyze different transmission line faults simultaneously in both time and frequency domains. The wavelet transform is useful in detecting and extracting faulty features of various types transmission line faults both in radial and non radial network because it is sensitive to signal irregularities but 
insensitive to the regular-like signal behavior. The wavelet transform can be interpreted as the inner product of the complex conjugate of wavelet function $\mathrm{h} * \mathrm{a}, \tau(\mathrm{t})$ and the input signal $\mathrm{s}(\mathrm{t})$

$\mathrm{WT}(\mathrm{a}, \tau)=\int \mathrm{h} * \mathrm{a}, \tau(\mathrm{t}) \cdot \mathrm{s}(\mathrm{t}) \mathrm{dt}$

(1)

Where the wavelet function $\mathrm{h}^{*} \mathrm{a}, \tau(\mathrm{t})$ is defined as

$\mathrm{h}^{*} \mathrm{a}, \tau(\mathrm{t})=\mathrm{a}^{-1 / 2} \mathrm{~h}(\mathrm{t}-\tau / \mathrm{a})$

The variable $\mathrm{a}$ is the scale parameter of the wavelet function and is proportional to the reciprocal of the frequency, $\tau$ is the translation parameter and $\mathrm{h}$ is called the mother wavelet. Wavelet analysis deals with unsteady signal while entropy expresses information of the signal. Hence Wavelet entropy can analyze fault signals more efficiently. The feature extraction is very important in signal processing operations because the rough and large data sets cause difficulties, when a network is trained. In this study half cycle of pre fault and half cycle of post fault of the faulty current signals of all the three phases are considered to reduce the data set in size.

Wavelet Packet is employed on all the current signals which are originated from EMTP simulation for obtaining high frequency detail component which gives distinctive features about the signals. A mother wavelet is a function that oscillates, has finite energy and zero mean value. Normally, the mother wavelet that resembles the analyzed signal is the best choice, as this would reduce the number of non-zero Wavelet coefficients. For our project purpose $\mathrm{DB}_{4}$ is selected as the mother wavelet. $\mathrm{DB}_{4}$ owns a good time resolution providing an accurate detection of the fast transients induced by faults.

In this paper wavelet coefficients of current signals are decomposed at level 3 for classification of fault types and identification of falty lines. Entropy values are considered as the input features for classification of different types of faults (SLG, LL, DLG and LLL) at different locations. Entropy is a common method in many fields, especially in signal processing applications. Entropy indicates the amount of information which is stored in observed signal. Wavelet packet is applied to find the entropy values of different current signals at different levels.

\section{Classification Of Faults USing ARTificial NeURal Network}

An artificial neural network can be defined as a data processing system consisting of a large number of simple, highly interconnected processing elements (artificial neurons) in an architecture inspired by the structure of the cerebral cortex of the human brain. ANN is powerful in pattern recognition, classification and generalization. Neural networks are useful for power system applications because they can be trained with off line data. Probabilistic neural networks (PNN) are a kind of radial basis network suitable for classification problems. The architecture is feed forward in nature which is similar to back propagation, but differs in the way that learning occurs. PNN is supervised learning algorithm but includes no weights in its hidden layer. Instead each hidden node represents an example vector, with the example acting as the weights to that hidden node. These are not adjusted at all. Figure III illustrates a sample PNN structure.

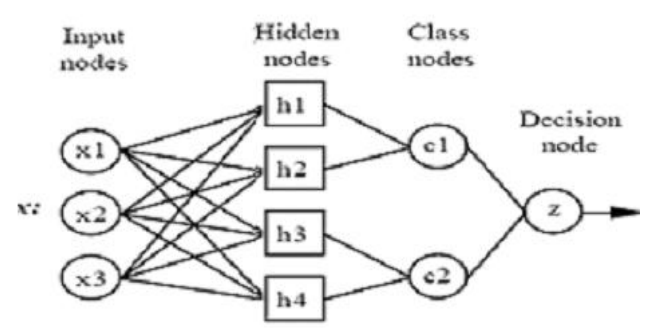

Figure III: PNN architecture

Basically, PNN consists of an input layer, which represents the input pattern or feature vector. The input layer is fully interconnected with the hidden layer, which consists of the example vectors (the training set for the PNN). The actual example vector serves as the weights as applied to the input layer. Finally, an output layer represents each of the possible classes for which the input data can be classified. However, the hidden layer is not fully interconnected to the output layer. The example nodes for a given class connect only to that class's output node and none other. The other important element of the PNN is the output layer and the determination of the class for which the input layer fits. This is done through a winner-takes-all approach. The output class node with the largest activation represents the winning class. While the class nodes are connected only to the example hidden nodes for their class, the input feature vector connects to all examples, and therefore influences their activations. Therefore the sum of the example vector activations determines the class of the input feature vector. In PNN algorithm, calculating the class-node activations is a simple process. For each class node, the example vector activations are summed, which are the sum of the products of the example vector and 
the input vector. The hidden node activation, shown in the following equation, is simply the product of the two vectors ( $\mathrm{E}$ is the example vector, and $\mathrm{F}$ is the input feature vector).

$\mathrm{H}_{\mathrm{i}}=\mathrm{E}_{\mathrm{i}} \mathrm{F}$

The class output activations are then defined as:

$C_{j}=\frac{\sum_{i=1}^{N} e^{\frac{h_{i}-1}{\gamma^{2}}}}{N}$

Where $\mathrm{N}$ is the total number of example vectors for this class $\mathrm{h}_{\mathrm{i}}$ is the hidden-node activation, and $\gamma$ is a smoothing factor. The smoothing factor is chosen through experimentation. If the smoothing factor is too large, details can be lost; again if the smoothing factor is too small, the classifier may not generalize well.

Total 30 sets of data, each set containing entropy values of the pure and ten types of faulty signals are created for the training and testing of the neural network for the non radial power system network to identify the fault types and faulty lines. Out of these 30 sets, 20 sets are used for the training purpose and remaining 10 sets of data are used for the testing purpose.

\section{IV.I TRAINING OF PNN}

The designed PNN model is trained by 1760 signals. The size of the input vector for type classification in non radial power system network is $9 \times 1760$; where 9 are approximation and detail values of level 1 and 3 for 3 phases and 1760 comes from 11 types of signals multiplied by 20 different locations in eight different lines.

The size of the input vector for faulty line identification is $9 \times 160$; where 9 are approximation and detail entropy values (one approximation value of level 3 and two detail values of level 1 and 3 for all the 3 phases) and 160 comes from 8 different lines multiplied by 20 different locations for each type of fault.

Target of PNN for type identification in radial and non radial power system networks are1, 2, 3, 4, 5, 6, 7, 8, 9, 10, 11 for Pure, SLG-R, SLG-Y, SLG-B, LL-R-Y, LL-Y-B, LL-B-R, LLG-R-Y, LLG-Y-B, LLG-B-R, LLL respectively.

For line identification in non radial power system networks the targets are 41, 42, 43, 44, 45, 46, 47 and 48 for line1, line2, line3, line4, line5, line6, line7, line8 respectively.

\section{IV.II TESTING OF PNN}

Pure signal along with ten different types of faults are considered in each set. In non radial network all the 11 types of waveforms are considered. These signals are taken in 10 different locations in 8 lines. Hence 80 different locations are made. 11 types of signals multiplied by 80 different locations give 880 different signals for non radial network for the testing of the PNN model for classification of faults.

Faulty line identification is performed in non radial network. 320 different signals are created for the testing of the PNN model for this purpose.

\section{Result}

After training, the neural network based fault detector and classifier is extensively tested using independent data sets consisting of fault scenarios not used previously for training the network. Fault type, fault location and fault resistances are changed for different faults in the validation/test data set to investigate the effects of these factors on the performance of the proposed method.

The results of classification for non radial system are shown in table I. To investigate the accuracy of the proposed method in these cases, 100\% accurate results are found for SLG and LLG type of faults. The accuracy rate is slightly decreased for some misclassifications in LL and LLL faults. In some cases three phase symmetrical faults are classified as line to line faults. Hence PNN model based fault identifier identifies the fault types with an accuracy of $98.40 \%$ in a very fast and effective manner. Fault resistances are varied to identify the fault types at different lines in non radial power system network. Fault resistances are varied from 0 to $30 \mathrm{ohms}$. Identification of faulty line is rigorously tested with current signals. Table II gives the classifier performance of faulty line identification of four different types of faults. $100 \%$ accurate results are found in line1, line5, line6, line7, line8. There are some mismatches in line2, 3 and 4. Hence PNN model based fault identifier identifies the faulty lines with an accuracy of $92.81 \%$. 
Wavelet Entropy and Neural Network Based Fault Detection on A Non Radial Power System Network

Table I: ANN result for fault classification in non radial system

\begin{tabular}{|c|c|c|c|c|c|c|c|c|c|c|c|}
\hline Type & Pure & LGR & LGY & LGB & LLRY & LLYB & LLBR & LLGRY & LLG YB & LLGBR & LLL \\
& $\mathbf{1}$ & $\mathbf{2}$ & $\mathbf{3}$ & $\mathbf{4}$ & $\mathbf{5}$ & $\mathbf{6}$ & $\mathbf{7}$ & $\mathbf{8}$ & $\mathbf{9}$ & $\mathbf{1 0}$ & $\mathbf{1 1}$ \\
\hline $\mathbf{1}$ & $\mathbf{8 0}$ & 0 & 0 & 0 & 0 & 0 & 0 & 0 & 0 & 0 & 0 \\
\hline $\mathbf{2}$ & 0 & $\mathbf{8 0}$ & 0 & 0 & 0 & 0 & 0 & 0 & 0 & 0 & 0 \\
\hline $\mathbf{3}$ & 0 & 0 & $\mathbf{8 0}$ & 0 & 0 & 0 & 0 & 0 & 0 & 0 & 0 \\
\hline $\mathbf{4}$ & 0 & 0 & 0 & $\mathbf{8 0}$ & 0 & 0 & 0 & 0 & 0 & 0 & 0 \\
\hline $\mathbf{5}$ & 0 & 0 & 0 & 0 & $\mathbf{7 5}$ & $\mathbf{2}$ & 0 & 0 & 0 & 0 & $\mathbf{3}$ \\
\hline $\mathbf{6}$ & 0 & 0 & 0 & 0 & 0 & $\mathbf{8 0}$ & 0 & 0 & 0 & 0 & 0 \\
\hline $\mathbf{7}$ & 0 & 0 & 0 & 0 & $\mathbf{1}$ & $\mathbf{3}$ & $\mathbf{7 6}$ & 0 & 0 & 0 & 0 \\
\hline $\mathbf{8}$ & 0 & 0 & 0 & 0 & 0 & 0 & 0 & $\mathbf{8 0}$ & 0 & 0 & 0 \\
\hline $\mathbf{9}$ & 0 & 0 & 0 & 0 & 0 & 0 & 0 & 0 & $\mathbf{8 0}$ & 0 & 0 \\
\hline $\mathbf{1 0}$ & 0 & 0 & 0 & 0 & 0 & 0 & 0 & 0 & 0 & $\mathbf{8 0}$ & 0 \\
\hline $\mathbf{1 1}$ & 0 & 0 & 0 & 0 & $\mathbf{2}$ & $\mathbf{2}$ & $\mathbf{1}$ & 0 & 0 & 0 & $\mathbf{7 5}$ \\
\hline
\end{tabular}

Table II: ANN result for faulty line identification in non radial system

\begin{tabular}{|c|c|c|c|c|c|c|c|c|}
\hline Location & Line 1 & Line 2 & Line 3 & Line 4 & Line 5 & Line 6 & Line 7 & Line 8 \\
& $\mathbf{4 1}$ & $\mathbf{4 2}$ & $\mathbf{4 3}$ & $\mathbf{4 4}$ & $\mathbf{4 5}$ & $\mathbf{4 6}$ & $\mathbf{4 7}$ & $\mathbf{4 8}$ \\
\hline $\mathbf{4 1}$ & $\mathbf{4 0}$ & 0 & 0 & 0 & 0 & 0 & 0 & 0 \\
\hline $\mathbf{4 2}$ & 0 & $\mathbf{3 2}$ & $\mathbf{5}$ & $\mathbf{3}$ & 0 & 0 & 0 & 0 \\
\hline $\mathbf{4 3}$ & 0 & $\mathbf{3}$ & $\mathbf{3 5}$ & 0 & $\mathbf{2}$ & 0 & 0 & 0 \\
\hline $\mathbf{4 4}$ & 0 & $\mathbf{4}$ & $\mathbf{4}$ & $\mathbf{3 0}$ & $\mathbf{2}$ & 0 & 0 & 0 \\
\hline $\mathbf{4 5}$ & 0 & 0 & 0 & 0 & $\mathbf{4 0}$ & 0 & 0 & 0 \\
\hline $\mathbf{4 6}$ & 0 & 0 & 0 & 0 & 0 & $\mathbf{4 0}$ & 0 & 0 \\
\hline $\mathbf{4 7}$ & 0 & 0 & 0 & 0 & 0 & 0 & $\mathbf{4 0}$ & 0 \\
\hline $\mathbf{4 8}$ & 0 & 0 & 0 & 0 & 0 & 0 & 0 & $\mathbf{4 0}$ \\
\hline \multicolumn{7}{|r|}{} \\
\hline
\end{tabular}

\section{Conclusions}

The purpose of this paper is to find an effective technique suitable for the fault classification of power system transmission lines. The proposed method uses wavelet packet decomposition which provides more features about the signal than classical wavelet. After wavelet packet decomposition of current signals up to three decomposition levels, the entropy values are calculated only for detail level 1 and 3 for each faulty current waveform. Therefore the size of the feature vectors is reduced considerably. The features obtained by this way are used as the inputs of ANN for determination of all types of faults along with affected phases within half cycle after the occurrence of the faults. The results presented in this paper confirm the possibility of developing an accurate fault classification and faulty line identification scheme that may aid the development of reliable transient-based protection schemes. Further investigations are being carried out to confirm the robustness of the performance under changes in the network configuration. The proposed scheme is easily comprehensible, deterministic and is feasible for practical implementation.

\section{ACKNOWLEDGMENT}

The authors acknowledge the support given by Techno India, Salt Lake, Kolkata, India for sponsoring this research.

\section{REFERENCES}

[1] S. El. Safty \& A. El-Zonkoly (2009), “Applying wavelet entropy principle in fault classification” Electrical Power and Energy Systems 31 pp.604-607

[2] Pradhan A. K. et Al. (Oct. 2004), "Wavelet-fuzzy Combined Approach for Fault Classification of a Series-Compensated Transmission Line" IEEE Trans. on Power Delivery, Vol. 19, No. 4.

[3] Jung. H et. al. (2007), "Novel Technique for Fault location estimation on parallel transmission lines using wavelet" Electrical Power and energy Systems 29 pp.76-82

[4] MATLAB 7.9.0 (R2009b) versionWavelet Toolbox, MathWorks Company.

[5] Kim. K.H. et al. (2007), "Wavelet and Neuro-Fuzzy Fault Location for Combined Transmission Systems" Electrical Power and Energy Systems 29 pp. $445-454$

[6] Chunju. F et Al. (2007), "Application of Wavelet Fuzzy Neural Network in Location Single Line to Ground Fault (SLG) in Distribution Lines" Electric Power and Energy Systems 29 pp. 497-503

[7] Eisa. A. Amir. A. \& Ramar. K, (2010) “Accurate one-end Fault location for Overhead Transmission Lines in Interconnected power system" Electrical Power and Energy Systems 32 pp. 383-389

[8] Salim. R.H et al. (April 2009), "Extended fault-Location Formulation for Power distribution Systems" IEEE Trans. on Power Delivery, Vol.24, No.2

[9] Borghetti. A et al. (2006), "On the Use of Continuous-Wavelet Transform for Fault Location in Distribution Power Systems" Electrical Power and Energy Systems 28 pp. 608-617 
[10] Fernando H. M. \& Abur. A. (October 1998), "Fault Location Estimation on Parallel Transmission Lines using Wavelet" IEEE Trans. on Power Delivery, Vol. 13, No 4.

[11] Saleh A. \& Al-jufout (2008), "Improved algorithm for Fault Simulation by hypothetical Stub moving along a medium-length transmission Line" ISSN 1450-216X Vol. 23, No 3 pp. 400-405

[12] Ekici S., Yildirim S., Poyraz M., (2008) "Entropy and energy based feature extraction for locating fault on transmission lines by using neural network and wavelet packet decomposition" Expert Systems with Applications 34 2937-2944

[13]. He, J.A. Starzyk, A self-organizing learning array system for power quality classification based onwavelet transform, IEEE Trans. PowerDeliv. 21 (1) (2006) 286-295

[14] Anamika Jain, V.S. Kale and A.S. Thoke, "Application of artificial neural networks to transmission line faulty phase selection and fault distance location," Proceedings of the IASTED International conference "Energy and Power System", Chiang Mai, Thailand, Mar. 29-31, 2006, pp. 262-267.

[15] D.Das N.K.Singh, A.K.Sinha, "A Comparison Of Fourier Transform And Wavelet Transform Methods For Detection And Classification Of Faults On Transmission Lines", in Proc. of IEEE Power India Conf., 2006.

[16] H.Khorashadi-Zadeh,"Artificial neural network approach to fault classification for double circuit transmission lines," in Proc. of IEEE Transmission and Distribution Conf., pp. 859-862, 2004.

[17] Xinzhou Dong, Wei Kong, Tao Cui, "Fault classification and faulted-phase selection based on the initial current traveling wave," IEEE Trans. Power Delivery, vol. 24, No.2, April 2009.

[18] A. Ngaopitakkal, W. Pongchaisrikul, A.Kundakorn, "Analysis of characteristics of simultaneous faults in electrical power system using wavelet transform," in Proc. IEEE International Conf. on Sustainable Energy Technologies, pp.249-252, 2008.

Soumyadip Jana obtained his B.Tech. \& M. Tech. from Bengal College of Engineering \& Technology, Durgapur and Netaji Subhash Engineering College, Garia, Kolkata respectively, in India . He is currently Assistant Professor in the Department of Electrical Engineering, Techno India, Salt Lake, West Bengal, India. His research interests include Power systems and application of artificial intelligence in power quality.

Gaurab Dutta obtained his Diploma and B.Tech from Ramakrishna Mission Shilpapitha, Belgharia and Netaji Subhash Engineering College, Garia, Kolkata respectively, in India. He worked as professional in the field of Electrical engineering at various industries and supervised projects from pre-commissioning level, namely National Electrical Engineering Co., Elex India Pvt. Ltd. and Hindustan National Glass, Sterling And Wilson Ltd. He is currently Technical Assistant in the Department of Electrical Engineering, Techno India, Salt Lake, West Bengal, India. His research interest includes Power systems. He is an authorized Electrical Supervisor by West Bengal Licensing Board Wiring for Systems not exceeding 650 volts and 250 volts for single phase. 